\title{
Diagnostic accuracy of auscultation and lung ultrasound in the assessment of adequate double lumen tube position for anesthetic trainee doctors - a single center, prospective study
}

\author{
Jeong Wook Park ${ }^{1,2}$, Ki Tae Jung ${ }^{1,3}$, Young Joon $\mathrm{Ki}^{1,2}$, Sang Hun $\mathrm{Kim}^{1,3}$ \\ ${ }^{1}$ Department of Anesthesiology and Pain Medicine, Chosun University Hospital, ${ }^{2}$ Department of Medicine, Graduate School of Chosun \\ University, ${ }^{3}$ Department of Anesthesiology and Pain Medicine, Chosun University, School of Medicine, Gwangju, Korea
}

Received November 11, 2020

Revised November 20, 2020

Accepted November 27, 2020

Corresponding author

Sang Hun Kim

Department of Anesthesiology and

Pain Medicine, Chosun University

School of Medicine, 309 Pilmun-

daero, Dong-gu, Gwangju 61453,

Korea

Tel: +82-62-220-3223

Fax: +82-62-223-2333

E-mail: ksh3223@chosun.ac.kr

ORCID:

https://orcid.org/0000-0003-3869-9470
Lung ultrasound can be used to assess adequate regional ventilation, similar to auscultation. Therefore, we evaluated whether the diagnostic accuracy of lung ultrasound was superior to that of auscultation in the assessment of proper double lumen tube (DLT) position, which were performed by anesthetic trainees. We conducted a single-center, prospective study of $69 \mathrm{pa}-$ tients. DLT insertion, auscultation, lung ultrasound, and fiberoptic bronchoscopy were sequentially conducted in the same patients in the supine and lateral positions. During lung ultrasound, the proper DLT position was defined when the lung pulse and barcode sign were visible on the non-ventilated lung, and lung sliding and seashore sign were observed in the ventilated lung. Fiberoptic bronchoscopy was performed for final verification of the DLT position as a standard test. Contingency tables were plotted to determine accuracy, sensitivity, specificity, positive predictive value (PPV), and negative predictive value (NPV) for each method. The primary outcome of this study was the accuracy of each method. Accuracy (60.9\%), sensitivity (100\%), specificity (12.9\%), PPV (58.5\%), and NPV (100\%) of ultrasound were equal to those of auscultation in the supine position. Accuracy (89.9\%), sensitivity (100\%), specificity (36.4\%), PPV (89.2\%), and NPV (100\%) of ultrasound were equal to that of auscultation in the lateral position. The prevalence of proper lung isolation was $55.1 \%$ in the supine position, and it increased to $84.1 \%$ in the lateral position. Lung ultrasound is not superior to auscultation for determination of the proper DLT position in both the supine and lateral positions.

Keywords: Accuracy; Auscultation; Double lumen tube; Fiberoptic bronchoscopy; Lung ultrasound

\section{INTRODUCTION}

One-lung ventilation (OLV) has been successfully achieved with double lumen tubes (DLTs) inz most thoracic or abdominal surgeries requiring lung isolation [1]. However, pre- cise positioning of DLT is essential for facilitating surgical exposure with successful lung isolation for thoracic surgery [1]. The auscultation method has been commonly used to evaluate regional ventilation and lung collapse, but it has low accuracy for confirmation of the optimal DLT position 
[2]. Therefore, direct visual confirmation using fiberoptic bronchoscopy (FOB) was considered a standard method [2]. Currently, a combination technique of auscultation and FOB is commonly used to determine adequate positioning of DLT. However, FOB examination is an invasive, time-consuming technique, and it requires an experienced operator to confirm the adequate DLT positioning. In addition, some institutions still do not perform FOB.

Recently, ultrasound examination has been used increasingly in various aspects of airway management. Ultrasound has been studied to evaluate its efficacy for determining adequate endotracheal intubation in the presence of an endotracheal cuff, airway extension, or lung-sliding sign in the thoracic region [3-6]. It has been suggested that ultrasound is useful to detect whether endotracheal intubation is adequate. Pneumothorax can also be diagnosed with high specificity and sensitivity based on lung ultrasound findings (absence of lung sliding and seashore sign with M-mode, or presence of lung pulse) [7-9]. Based on the concept that the non-ventilated thoracic condition after OLV is similar to that with pneumothorax, the proper lung isolation was evaluated with ultrasound findings used in patients with pneumothorax. Several investigators have suggested that lung ultrasound is a noninvasive, effective, and simple technique that is superior to auscultation in determining the proper DLT position, but both methods were not ensured in confirming the misplacement [10-13]. However, there is still a lack of evidence for the usefulness of lung ultrasound in determining proper DLT positions.

This study compared the diagnostic accuracy of auscultation and lung ultrasound in the assessment of proper DLT position, which anesthetic trainee doctors performed in patients who underwent thoracic surgeries. The primary outcome of this study was the overall accuracy of each method.

\section{MATERIALS AND METHODS}

This prospective, randomized, controlled, and investigator-blinded study was approved by the Institutional Review Board of Chosun University Hospital (Chosun 2018-11-008001) and was registered with the Clinical Research Information Service (CRIS: https://cris.nih.go.kr/, ref: KCT0003881) on May 3, 2019. This study was conducted according to the Declaration of Helsinki 1964 and all subsequent revisions.
The subjects included patients aged over 18 years with an American Society of Anesthesiologists (ASA) physical status I-III and who were scheduled to undergo elective thoracic surgery requiring one-lung ventilation with DLT. We excluded patients $<18$ years of age, those with anticipated difficult airways or a tracheostomy tube in situ, and those diagnosed with pleural effusion, pleurodesis or post-inflammatory pleural adhesions, chronic obstructive pulmonary disease, or mesothelioma.

Patients were screened a day before surgery, and written informed consent was obtained from all participants or a legal surrogate, after a thorough explanation of the purpose of this study. A total of 69 patients were included in the study from August 6, 2019 to April 13, 2020, but they were not randomized.

The attending anesthesiologist was responsible for obtaining consent, managing anesthesia, and determining the size and side of DLT (Mallinckrodt Endobronchial Tube, Covidien, USA). After premedication with intramuscular midazolam $(0.05 \mathrm{mg} / \mathrm{kg})$, the patients were transported to an operating room. Before the anesthesia was induced, the principal investigator (PI) or other investigators evaluated whether the patients had intact breathing sounds and lung sliding. We excluded patients without lung sliding before anesthesia induction. They did not participate in the auscultation, lung ultrasound, or FOB. Auscultation and lung ultrasound were performed at the same points as follows: 1) the point at which the second intercostal space meets the midclavicular line and 2) the point at which the fifth intercostal space meets the midclavicular line.

Following total intravenous anesthesia induction with propofol, remifentanil, and rocuronium, the attending anesthesiologist performed bronchial intubation and recorded the initial intubation depths at the central incisor level. During two-lung ventilation (TLV), the tidal volume was 8 $\mathrm{mL} / \mathrm{kg}$ (ideal body weight), with the oxygen-air mixture in a 1:1 ratio. During OLV, the tidal volume was $6 \mathrm{~mL} / \mathrm{kg}$ with $100 \%$ oxygen. The end-tidal carbon dioxide and oxygen saturation were maintained between 30 and $35 \mathrm{mmHg}$ and 93-100\%, respectively.

In the supine position, auscultation, lung ultrasound, and FOB were performed sequentially to assess whether the DLT position was proper. The investigators were randomly assigned as the examiner of either auscultation, lung ultra- 
sound, or FOB. Randomization was performed using a computer-generated random table with the randomly permuted block method (1:1:1 allocation ratio).

First, auscultation was performed as follows: 1) at both lung points during TLV, 2) at the points of the ventilated lung during OLV, and 3) at non-ventilated lung points during OLV. We defined the proper DLT position as follows: intact breathing sound at two points of the ventilated lung, and absence of breathing sound at two points in the non-ventilated lung.

Next, lung ultrasound examiners performed the examination of lung ultrasound during OLV using an M-Turbo Ultrasound system (SonoSite Ltd., UK) with a 13-6 MHz linear transducer. We placed this transducer sagittal at predefined measurement points in both midclavicular lines. If lung sliding with $2 \mathrm{D}$ mode and seashore sign with $\mathrm{M}$-mode were shown in the non-ventilated lung, we defined it as an improper DLT position. If the lung pulse with 2D mode and barcode sign with M-mode were visible, it was defined as the proper DLT position.

Finally, FOB examiners determined whether the proximal part of the blue cuff was located just below the carina without obstruction of the opposite bronchus (proper DLT position), with FOB (intubation fiberscope 3.7×65, Karl Storz, Germany). If the blue cuff of the DLT was located deep in the main bronchus, we defined it as a suboptimal position. If the blue cuff of the DLT was not located in the main bronchus of the nonsurgical lung or in the trachea, we defined it as malposition.

After FOB, the principal investigator adjusted the depth of DLT with FOB results and recorded the adjusted intubation depths. The principal investigator gathered all data reported as 'correct' or 'incorrect' from each investigator in the case record form, and each investigator did not share results until the final analysis was completed. After the lateral decubitus position, this evaluation process was repeated in the non-ventilated lung during OLV.

We recorded demographic data, such as age, sex, height, weight, body mass index (BMI), ASA physical status, diagnosis, side of DLT, and type of operation. We calculated the true positive (TP), true negative (TN), false positive (FP), and false negative (FN), sensitivity, specificity, positive predictive value (PPV), negative predictive value (NPV), and accuracy of each method, with fiberoptic bronchoscopy findings as standard test results. Sensitivity, specificity, PPV, NPV, and accuracy were calculated as follows: Sensitivity (\%)=TP/(TP+ $\mathrm{FN}) \times 100$, specificity $(\%)=\mathrm{TN} /(\mathrm{TN}+\mathrm{FP}) \times 100, \mathrm{PPV}(\%)=\mathrm{TP} /(\mathrm{TP}$ $+\mathrm{FP}) \times 100$, NPV $(\%)=\mathrm{TN} /(\mathrm{TN}+\mathrm{FN}) \times 100$, and accuracy $(\%)=$ $(\mathrm{TP}+\mathrm{TN}) /(\mathrm{TP}+\mathrm{TN}+\mathrm{FP}+\mathrm{FN}) \times 100$.

\section{Statistical analysis}

The primary outcomes of this study were accuracy of auscultation and lung ultrasound to determine the proper DLT position. SPSS software (Windows ver. 26.0, IBM Corp., Armonk, NY, USA) was used for all statistical analyses, which were performed by an independent researcher who did not participate in the examinations and data collection.

Based on a previous report, $[10,13,14]$ we assumed that lung ultrasound would be superior to auscultation to determine the proper DLT position. We calculated the sample size with a significance level $(\alpha)$ of $5 \%$; power $(1-\beta)$, $90 \%$; expected accuracy of auscultation, $60 \%$; and expected accuracy of lung ultrasound, $85 \%$, via a website for sample size calculation (https://www.sealedenvelope.com/power/binary-superiority/). We finally decided on a sample size of 69 patients, considering a dropout rate of approximately $10 \%$, because we designed the study with the sequential tests in the single group.

All data are presented as means $(95 \%$ confidence intervals [CI]), number (percentage) of patients (n [\%]), or percentage (\%). An analysis of the contingency table was used to calculate the accuracy, sensitivity, specificity, PPV, and NPV for each method. The contingency table data were analyzed using the chi-square test or Fisher's exact test, and were also analyzed with McNemar's test to examine the extent of disagreement between each method. p values $<0.05$ were considered statistically significant.

\section{RESULTS}

A total of 69 patients were finally enrolled in both auscultation and lung ultrasound groups (Fig. 1). Table 1 shows the demographic data of the participating patients.

The main results of this study were as follows: accuracy, sensitivity, specificity, PPV, and NPV of ultrasound were equal to that of auscultation, regardless of the position change. However, the accuracy, specificity, and PPV of both methods were increased in the lateral decubitus position 


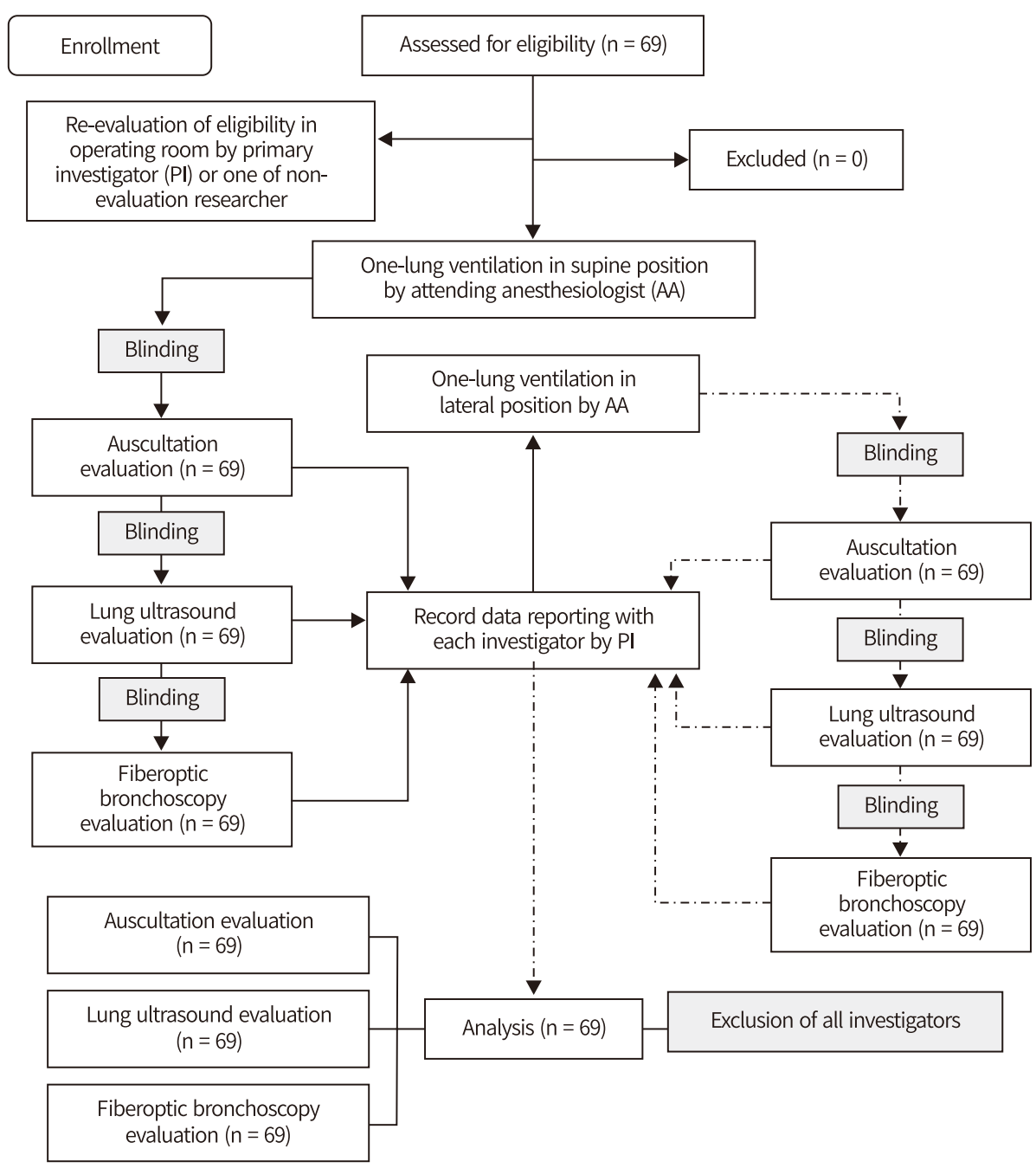

Fig. 1. CONSORT diagram for patient recruitment.

Table 1. Demographic data

\begin{tabular}{lclc}
\hline \multicolumn{1}{c}{ Variables } & Cohort $(\mathrm{n}=69)$ & \multicolumn{1}{c}{ Variables } & Cohort $(\mathrm{n}=69)$ \\
\hline Age $(\mathrm{yr})$ & $46.3(41.5-51.2)$ & Types of surgeries & \\
Sex $(\mathrm{M} / \mathrm{F})$ & $15(21.7) / 54(78.3)$ & Biopsy & $9(13.0)$ \\
Height $(\mathrm{cm})$ & $168.1(165.8-170.4)$ & Lobectomy & $12(17.4)$ \\
Weight $(\mathrm{kg})$ & $61.3(58.8-63.8)$ & Mass removal & $2(2.9)$ \\
BMI $\left(\mathrm{kg} / \mathrm{m}^{2}\right)$ & $21.6(21.0-22.3)$ & Rib fixation & $6(8.7)$ \\
ASA-PS & & Sympathectomy & $4(5.8)$ \\
I & $30(43.5)$ & Wedge resection & $36(52.2)$ \\
II & $36(52.2)$ & Diagnosis & \\
III & $3(4.3)$ & Chest wall mass & $1(1.4)$ \\
Side of DLT used & & Hyperhidrosis & $4(5.8)$ \\
Left DLT & $66(95.7)$ & Lung mass & $22(31.9)$ \\
Right DLT & $3(4.3)$ & Pneumothorax & $35(50.7)$ \\
& & Rib fracture & $6(8.7)$ \\
& & Thymoma & $1(1.4)$ \\
\hline
\end{tabular}

Values are expressed as mean (95\% confidence intervals) or number (percentage) of patients. ASA-PS: American Society of Anesthesiologists physical status, BMI: body mass index, DLT: double lumen tube. after correction of DLT depth according to FOB findings, as shown in Tables 2, 3, and 4.

In determining the appropriate DLT position in a supine position, the accuracy of lung ultrasound was equal to that of auscultation, which was 60.9\% (48.4\%-72.4\%) (Table 4). The sensitivity and specificity of lung ultrasound were equal to those of auscultation, which were $100 \%$ (88.6\%-100.0\%) and $12.9 \%(4.2 \%-30.8 \%$ ), respectively (Table 4). The PPV and NPV of lung ultrasound were equal to those of auscultation, which were $58.5 \%(45.6 \%-70.3 \%)$ and 100\% (39.6\%-100.0\%), respectively (Table 4).

In determining the appropriate DLT position in a lateral position, the accuracy of lung ultrasound was equal to that of auscultation, which was $89.9 \%$ (80.2\%-95.8\%) (Table 4). The sensitivity and specificity of lung ultrasound were equal to those of auscultation, which were $100 \%$ (92.3\%-100.0\%) and 36.4\% (12.4\%-68.4\%), respectively (Tables 2-4). The PPV 
Table 2. Contingency table for auscultation and lung ultrasound

\begin{tabular}{|c|c|c|c|c|c|c|c|}
\hline \multirow{2}{*}{\multicolumn{2}{|c|}{ Group }} & \multicolumn{2}{|c|}{ DLT position by FOB in supine position } & \multirow{2}{*}{$p$ values } & \multicolumn{2}{|c|}{ DLT position by FOB in lateral position } & \multirow{2}{*}{$p$ values } \\
\hline & & Correct & Incorrect & & Correct & Incorrect & \\
\hline \multirow[t]{2}{*}{ Auscultation } & Correct & $38(55.1)$ & $27(39.1)$ & $0.316^{*},<0.001^{\dagger}$ & $58(84.1)$ & $7(10.1)$ & $<0.001^{*}, 0.016^{\dagger}$ \\
\hline & Incorrect & $0(0)$ & $4(5.8)$ & & $0(0)$ & $4(5.8)$ & \\
\hline \multirow[t]{2}{*}{ Lung ultrasound } & Correct & $38(55.1)$ & $27(39.1)$ & $0.316^{*},<0.001^{\dagger}$ & $58(84.1)$ & $7(10.1)$ & $<0.001^{*}, 0.016^{\dagger}$ \\
\hline & Incorrect & $0(0)$ & $4(5.8)$ & & $0(0)$ & $4(5.8)$ & \\
\hline
\end{tabular}

Values are expressed as number (percentage) of patients $n$ (\%). DLT: double lumen tube, FOB: fiberoptic bronchoscopy. ${ }^{*}$ Analysis using Fisher's exact test. ${ }^{\dagger}$ Analysis using McNemar's test.

Table 3. Correlated proportion comparison of lung ultrasound to auscultation for DLT position

\begin{tabular}{c}
$\begin{array}{c}\text { Lung ultrasound } \\
(\mathrm{n}=69)\end{array} \quad$ p value \\
\cline { 2 - 2 } Correct Incorrect \\
\hline
\end{tabular}

\begin{tabular}{clcll}
\hline $\begin{array}{c}\text { Supine position } \\
\begin{array}{c}\text { Auscultation } \\
(\mathrm{n}=69)\end{array}\end{array}$ & Correct & $65(94.2)$ & $0(0)$ & $<0.001^{*}, 1.000^{\dagger}$ \\
$\begin{array}{c}\text { Lateral position } \\
\text { Incorrect }\end{array}$ & $0(0)$ & $4(5.8)$ & \\
$\begin{array}{c}\text { Auscultation } \\
(\mathrm{n}=69)\end{array}$ & Correct & $65(94.2)$ & $0(0)$ & $<0.001^{*}, 1.000^{\dagger}$ \\
& Incorrect & $0(0)$ & $4(5.8)$ &
\end{tabular}

Values are expressed as number (percentage) of patients $n$ (\%). DLT: double lumen tube. ${ }^{*}$ Analysis using Fisher's exact test. ${ }^{\dagger}$ Analysis using McNemar's test.

and NPV of lung ultrasound were equal to those of auscultation, which were $89.2 \%(78.5 \%-95.2 \%)$ and $100 \%(39.6 \%-$ 100.0\%), respectively (Tables 2-4).

Table 2 shows a statistically significant difference in the contingency table for auscultation and lung ultrasound in the supine and lateral positions, with fiberoptic bronchoscopy as the standard test. The auscultation and lung ultrasound showed a statistically significant disagreement with FOB $(\mathrm{p}<0.001$ in the supine position and $\mathrm{p}=0.016$ in the lateral position, Table 2), but lung ultrasound did not show a statistically significant disagreement with auscultation in either the supine or lateral positions ( $p=1.000$ in both the supine and lateral positions, Table 3).

The prevalence of proper lung isolation was $55.1 \%$ $(42.7 \%-66.9 \%)$ in the supine position, and it increased to $84.1 \%(72.8 \%-91.4 \%)$ in the lateral decubitus position after correction of DLT depth according to FOB findings (Tables 2, 5).

Adjustments in the DLT position were required because of either suboptimal position with OLV or malposition with
Table 4. Accuracy, sensitivity, specificity, PPV, and NPV of auscultation and lung ultrasound in determining proper DLT positioning

\begin{tabular}{lcccc}
\hline \multirow{2}{*}{ Outcomes } & \multicolumn{2}{c}{ Auscultation $(\mathrm{n}=69)$} & \multicolumn{2}{c}{ Lung ultrasound $(\mathrm{n}=69)$} \\
\cline { 2 - 5 } & Value $(\%)$ & $95 \% \mathrm{Cl}$ & Value $(\%)$ & $95 \% \mathrm{Cl}$ \\
\hline Supine position & & & & \\
Accuracy & 60.9 & $48.4-72.4$ & 60.9 & $48.4-72.4$ \\
Sensitivity & 100.0 & $88.6-100.0$ & 100.0 & $88.6-100.0$ \\
Specificity & 12.9 & $4.2-30.8$ & 12.9 & $4.2-30.8$ \\
PPV & 58.5 & $45.6-70.3$ & 58.5 & $45.6-70.3$ \\
NPV & 100.0 & $39.6-100.0$ & 100.0 & $39.6-100.0$ \\
Lateral position & & & & \\
Accuracy & 89.9 & $80.2-95.8$ & 89.9 & $80.2-95.8$ \\
Sensitivity & 100.0 & $92.3-100.0$ & 100.0 & $92.3-100.0$ \\
Specificity & 36.4 & $12.4-68.4$ & 36.4 & $12.4-68.4$ \\
PPV & 89.2 & $78.5-95.2$ & 89.2 & $78.5-95.2$ \\
NPV & 100.0 & $39.6-100.0$ & 100.0 & $39.6-100.0$ \\
\hline
\end{tabular}

Values are expressed as percentage 95\% confidence intervals (CI). NPV: negative predictive value, PPV: positive predictive value.

Table 5. Analysis of proper DLT position depending on patient positions

\begin{tabular}{lcc}
\hline & $\begin{array}{c}\text { Supine } \\
\text { position }\end{array}$ & $\begin{array}{c}\text { Lateral } \\
\text { position* }\end{array}$ \\
\hline Correct position (No change) & $38(55.1)$ & $58(84.1)$ \\
Incorrect position & $31(44.9)$ & $11(15.9)$ \\
Causes of incorrect position & & \\
$\quad \begin{array}{l}\text { Suboptimal position (One-lung ventilation) } \\
\quad \text { Malposition (Two-lung ventilation) }\end{array}$ & $28(40.6)$ & $6(8.7)$ \\
\hline
\end{tabular}

Values are expressed as number or number (percentage) of patients. DLT: double lumen tube, FOB: fiberoptic bronchoscopy. *Lateral position changed after correction of DLT depth according to FOB findings.

TLV (Table 5). The suboptimal position and the malposition were $40.6 \%$ and $4.3 \%$ of patients in the supine position, whereas they were $8.7 \%$ and $7.2 \%$ of patients in the lateral position. Both auscultation and lung ultrasound did not reveal an inappropriate DLT position in 39\% of patients in 
a supine position, and in $10 \%$ of patients in the lateral position (Table 2).

\section{DISCUSSION}

This study compared the diagnostic accuracy of lung ultrasound and auscultation, which anesthetic trainee doctors performed for the determination of proper DLT positions in patients undergoing thoracic surgeries. We demonstrated that lung ultrasound was not superior to auscultation, with equal diagnostic accuracy in the supine (60.9\%) and lateral positions (89.9\%). In the lateral position after correction of DLT depth according to FOB findings in a supine position, the specificity, PPV, and accuracy were increased, but the sensitivity and NPV did not change.

We commonly evaluate the proper DLT position with two steps: confirmation of regional ventilation distribution by auscultation (functional lung isolation) and precise DLT position with FOB (anatomical lung isolation) [11]. However, previous studies showed a wide range of sensitivity (47\%$100 \%$ ), low sensitivity (18\%-56\%), and moderate accuracy (42\%-70\%) [10-14]. Furthermore, de Bellis et al. [15] showed that the misplacement and critical malposition of DLT was confirmed in $32 \%$ and $5 \%$ of patients with FOB, even though the anesthesiologist considered to be in a proper DLT position under the clinical evaluation in a supine position. This study also showed $100 \%$ sensitivity, $13 \%$ specificity, and $61 \%$ accuracy in the supine position. In addition, the misplacement and critical malposition of DLT was confirmed in $40.6 \%$ and $4.3 \%$ of patients with FOB. Therefore, our results support the evidence that auscultation is unreliable for choosing the proper DLT position, and FOB is frequently required for adequate placement $[2,15]$. Ultimately, FOB is required for confirmation of proper placement of DLTs if available. However, in the situation where the FOB cannot be available, we do not have a choice to rely on auscultation.

Recently, lung ultrasound has been suggested as a useful tool in airway management to confirm the correct position of endotracheal tubes, and it has also been a method to confirm adequate regional ventilation similar to auscultation [16-19]. However, a few studies have been conducted to evaluate the efficacy of lung ultrasound to confirm the proper DLT positions for OLV [10-14]. These studies sug- gested that lung ultrasound was superior to auscultation in the determination of DLT position, with a more concise range of sensitivity (70\%-95\%), higher specificity (55.6\%94.7\%), and higher accuracy (71\%-89\%) compared with those of auscultation $[10,11,13]$. In addition, the combination of lung ultrasound and clinical assessment was useful to ensure more precise DLT placement than with clinical assessment alone $[12,14]$. However, this study did not show the superiority of lung ultrasound over auscultation in the supine position, in which the sensitivity, specificity, and accuracy of lung ultrasound were equal to those of auscultation. Compared with previous studies, this study showed lower specificity and accuracy.

Even though the DLT position has been optimized in the supine position by correction according to FOB findings, the optimized DLT positions may be changed after moving from the supine to the lateral position. Therefore, we evaluated the proper DLT position using auscultation and lung ultrasound in both the supine and lateral positions. Hu et al. [13] showed that the overall sensitivity and accuracy of lung ultrasound decreased after moving from the supine to the lateral position, while the overall specificity was increased. However, the positioning of the left and right DLTs differs significantly due to anatomical differences. Hu et al. [13] showed that the accuracy and sensitivity were increased in patients with the left DLT, and the specificity was decreased, while they were opposite in patients with right DLT. This study showed that the overall accuracy increased in the lateral position after the readjustment of DLT depth according to FOB findings. This discrepancy can be explained by the different proportions of right and left DLTs. In this study, 4.3\% of enrolled patients had right-sided DLT, while the study by Hu et al. [13] consisted of $44.7 \%$ of patients with right-sided DLT.

During the lung ultrasound evaluation, lung sliding and lung pulse are commonly used to distinguish the adequately ventilated lung with a homogenous movement below the pleural line [20-23]. In particular, lung pulses have been demonstrated to have high sensitivity (93\%) and specificity (100\%) as a sign for adequate lung isolation in a non-ventilated lung [20,24]. While the ventilated lung shows lung sliding in B-mode and sea-shore sign in M-mode, the non-ventilated lung shows the lung pulse (pulsatile movement of the lung) in B-mode and bar-code sign in M-mode [24,25]. 
Therefore, an adequate DLT position can be assumed if there is lung sliding in the ventilated lung and lung pulse in the non-ventilated lung. In this study, we used these two ultrasound modes (B-mode and $\mathrm{M}$-mode) to confirm lung ventilation. We first confirmed lung ventilation with $B$-mode and finally with $\mathrm{M}$-mode because the examiners were the anesthetic trainee doctors and the $\mathrm{M}$-mode was easier than the B-mode to confirm ventilation for beginners.

Overall examination of lung ultrasound also requires a learning curve of 7 months with a linear trend for reporting accuracy and quality of lung ultrasound [26]. However, Rouby et al. [27] suggested that trainees without expertise in lung ultrasound acquired skills in lung ultrasound to monitor lung aeration, with a rapid learning curve as 25 ultrasound examinations under the supervision of expert anesthesiologists in lung ultrasound. In particular, skills in lung ultrasound can be easily acquired and retained for a long time by a brief tutorial to determine the presence or absence of specific sonographic findings such as lung sliding and lung pulse sign [28]. Therefore, we first trained lung ultrasound and FOB investigators for 6 months before the enrollment of the first patient.

There are a few limitations to this study. First, instead of randomization of patients in two groups, we conducted auscultation, lung ultrasound, and FOB in the same patients (single group). Second, the choice of optimal DLT size is important to reduce the displacement or the malposition of DLTs because the inappropriately sized DLTs can be placed into the main bronchus too far distally or proximally. However, we did not record or analyze this data, even though we used 35 Fr, 37 Fr, and 39 Fr DLTs. Finally, lung ultrasound is unreliable in cases that can abolish the lung sliding sign, such as in patients with surgical pleurodesis or post-inflammatory pleural adhesions, chronic obstructive pulmonary disease, or mesothelioma [11]. Such conditions must be taken into account before using lung ultrasound. In this study, we excluded these patients during preanesthetic evaluation and by rechecking the absence of lung sliding before anesthesia induction. Therefore, further studies are required for the evaluation of lung ultrasound to determine the proper DLT position in patients with conditions excluded in this study.

In conclusion, we cannot suggest that lung ultrasound is superior to auscultation for determination of the proper DLT position because of the equal results of accuracy, sensitivity, specificity, PPV, and NPV in both the supine and lateral positions. Lung ultrasound has high sensitivity to detect the proper DLTs, but low sensitivity to detect the misplacement of DLTs in both positions. Changing to the lateral position after adjustment of intubation depth with FOB findings in the supine position increased the accuracy of both methods, but it increased the malposition of DLTs while decreasing the misplacement of DLTs. Therefore, we additionally recommend that FOB reassessment be performed to reduce the risk of DLT malposition.

\section{ACKNOWLEDGMENTS}

This study was supported by research funds from Chosun University (Donggu, Gwangju, Republic of Korea), 2019. Chosun University had no role in the design or conduct of the study; collection, management, analysis, and interpretation of the data; and preparation, review, or approval of the manuscript.

We would like to thank Dr. Bo Hyun Jang and Dr. Su Yeon Jo for their assistance in evaluating auscultation, lung ultrasound, and fiberoptic bronchoscopy.

\section{CONFLICT OF INTEREST}

No potential conflict of interest relevant to this article was reported.

\section{REFERENCES}

1. Campos JH. Current techniques for perioperative lung isolation in adults. Anesthesiology 2002;97:1295-301.

2. Alliaume B, Coddens J, Deloof T. Reliability of auscultation in positioning of double-lumen endobronchial tubes. Can J Anaesth 1992;39:687-90.

3. Abbasi S, Farsi D, Zare MA, Hajimohammadi M, Rezai M, Hafezimoghadam P. Direct ultrasound methods: a confirmatory technique for proper endotracheal intubation in the emergency department. Eur J Emerg Med 2015;22:10-6.

4. Tessaro MO, Salant EP, Arroyo AC, Haines LE, Dickman E. Tracheal rapid ultrasound saline test (T.R.U.S.T.) for confirming correct endotracheal tube depth in children. Resuscitation 2015;89:8-12.

5. Hoffmann B, Gullett JP, Hill HF, Fuller D, Westergaard MC, Hosek WT, et al. Bedside ultrasound of the neck confirms 
endotracheal tube position in emergency intubations. Ultraschall Med 2014;35:451-8.

6. Ahn JH, Kwon E, Lee SY, Hahm TS, Jeong JS. Ultrasoundguided lung sliding sign to confirm optimal depth of tracheal tube insertion in young children. Br J Anaesth 2019;123:30915.

7. Lichtenstein DA, Mezière G, Lascols N, Biderman P, Courret JP, Gepner A, et al. Ultrasound diagnosis of occult pneumothorax. Crit Care Med 2005;33:1231-8.

8. Husain LF, Hagopian L, Wayman D, Baker WE, Carmody KA. Sonographic diagnosis of pneumothorax. J Emerg Trauma Shock 2012;5:76-81.

9. Volpicelli G. Sonographic diagnosis of pneumothorax. Intensive Care Med 2011;37:224-32.

10. Álvarez-Díaz N, Amador-García I, Fuentes-Hernández M, Dorta-Guerra R. Comparison between transthoracic lung ultrasound and a clinical method in confirming the position of double-lumen tube in thoracic anaesthesia. A pilot study. Rev Esp Anestesiol Reanim 2015;62:305-12.

11. Parab SY, Kumar P, Divatia JV, Sharma K. A prospective randomized controlled double-blind study comparing auscultation and lung ultrasonography in the assessment of double lumen tube position in elective thoracic surgeries involving one lung ventilation at a tertiary care cancer institute. Korean J Anesthesiol 2019;72:24-31

12. Sustić A, Protić A, Cicvarić T, Zupan Z. The addition of a brief ultrasound examination to clinical assessment increases the ability to confirm placement of double-lumen endotracheal tubes. J Clin Anesth 2010;22:246-9.

13. Hu WC, Xu L, Zhang Q, Wei L, Zhang W. Point-of-care ultrasound versus auscultation in determining the position of double-lumen tube. Medicine (Baltimore) 2018;97:e9311.

14. Parab SY, Divatia JV, Chogle A. A prospective comparative study to evaluate the utility of lung ultrasonography to improve the accuracy of traditional clinical methods to confirm position of left sided double lumen tube in elective thoracic surgeries. Indian J Anaesth 2015;59:476-81.

15. de Bellis M, Accardo R, Di Maio M, La Manna C, Rossi GB, Pace MC, et al. Is flexible bronchoscopy necessary to confirm the position of double-lumen tubes before thoracic surgery? Eur J Cardiothorac Surg 2011;40:912-6.

16. Das SK, Choupoo NS, Haldar R, Lahkar A. Transtracheal ultrasound for verification of endotracheal tube place- ment: a systematic review and meta-analysis. Can J Anaesth 2015;62:413-23.

17. Blaivas M, Tsung JW. Point-of-care sonographic detection of left endobronchial main stem intubation and obstruction versus endotracheal intubation. J Ultrasound Med 2008;27:7859.

18. Pfeiffer P, Bache S, Isbye DL, Rudolph SS, Rovsing L, Børglum J. Verification of endotracheal intubation in obese patients - temporal comparison of ultrasound vs. auscultation and capnography. Acta Anaesthesiol Scand 2012;56:571-6.

19. Pfeiffer P, Rudolph SS, Børglum J, Isbye DL. Temporal comparison of ultrasound vs. auscultation and capnography in verification of endotracheal tube placement. Acta Anaesthesiol Scand 2011;55:1190-5.

20. Lichtenstein DA. BLUE-protocol and FALLS-protocol: two applications of lung ultrasound in the critically ill. Chest 2015;147:1659-70.

21. Khosla R. Bedside lung ultrasound in emergency (BLUE) protocol: a suggestion to modify. Chest 2010;137:1487; author reply $1487-8$.

22. Lichtenstein D. Lung ultrasound in acute respiratory failure an introduction to the BLUE-protocol. Minerva Anestesiol 2009;75:313-7.

23. Lichtenstein DA, Mezière GA. Relevance of lung ultrasound in the diagnosis of acute respiratory failure: the BLUE protocol. Chest 2008;134:117-25.

24. Lichtenstein DA, Lascols N, Prin S, Mezière G. The "lung pulse": an early ultrasound sign of complete atelectasis. Intensive Care Med 2003;29:2187-92.

25. Alerhand S, Tsung JW. Unmasking the lung pulse for detection of endobronchial intubation. J Ultrasound Med 2020;39:2105-9.

26. Tutino L, Cianchi G, Barbani F, Batacchi S, Cammelli R, Peris A. Time needed to achieve completeness and accuracy in bedside lung ultrasound reporting in intensive care unit. Scand J Trauma Resusc Emerg Med 2010;18:44.

27. Rouby JJ, Arbelot C, Gao Y, Zhang M, Lv J, An Y, et al. Training for lung ultrasound score measurement in critically ill patients. Am J Respir Crit Care Med 2018;198:398-401.

28. Lyon M, Walton P, Bhalla V, Shiver SA. Ultrasound detection of the sliding lung sign by prehospital critical care providers. Am J Emerg Med 2012;30:485-8. 